\title{
Ti/TaN Bilayer for Efficient Injection and Reliable AlGaN Nanowires LEDs
}

\author{
Davide Priante ${ }^{1}$, Bilal Janjua ${ }^{1}$, Aditya Prabaswara ${ }^{1}$, Ram Chandra Subedi ${ }^{1}$, Rami T. Elafandy ${ }^{1}$, Sergei \\ Lopatin $^{2}$, Dalaver H. Anjum ${ }^{2}$, Chao Zhao ${ }^{1}$, Tien Khee $\mathrm{Ng}^{1}$, Boon S. Ooi ${ }^{1 *}$ \\ ${ }^{I}$ King Abdullah University of Science and Technology (KAUST), Photonics Laboratory, Thuwal 23955-6900, Saudi Arabia \\ ${ }^{2}$ King Abdullah University of Science and Technology (KAUST), Imaging and Characterization Core Lab, Thuwal 23955-6900, Saudi Arabia \\ *e-mail address: boon.ooi@kaust.edu.sa
}

\begin{abstract}
Reliable operation of UV AlGaN-based nanowires-LED at high injection current was realized by incorporating a Ti-pre-orienting/TaN-diffusion-barrier bilayer, thus enhancing external quantum efficiency, and resolving the existing device degradation issue in group-III-nanowireson-silicon devices.

OCIS codes: (160.6000) Semiconductor materials; (250.5590) Quantum-well, -wire and -dot devices
\end{abstract}

\section{Introduction}

AlGaN ternary compounds are wavelength tunable in the UV-C $(\sim 200 \mathrm{~nm})$ to UV-A $(\sim 360 \mathrm{~nm})$ regime. The materials, being inert and environmental friendly, are promising for a large variety of application such as water purification, chemical agent detection and lighting. Despite its technological infancy as compared to planar AlGaN LEDs, nanowires (NWs) devices when realized on silicon substrate offer economy of scale and a great potential for industry adoption. However, it is noted that silicon suffers from the spontaneous formation of insulating $\mathrm{SiN}_{\mathrm{x}}$ at the NWs/substrate interface.[1] Moreover, the band discontinuity at $\mathrm{GaN} / \mathrm{SiN}_{\mathrm{x}} / \mathrm{Si}$ interface is undesirable for efficient device performance. In this regard, III-nitride NWs grown on pre-orienting thin film metal layer have been demonstrated.[2, 3] However, the high thermal mismatch between metals, e.g. Ti, and $\mathrm{Si}$, causes delamination of the Ti thin-film from the Si substrate when devices are operated at elevated injection current density, thus causing device failure. In addition, the resultant surface roughness affects the effective planarization of devices[4]. We implemented $\mathrm{Al}_{\mathrm{x}} \mathrm{Ga}_{1-\mathrm{x}} \mathrm{N}$ (quantum disk)/ $\mathrm{Al}_{\mathrm{y}} \mathrm{Ga}_{1-\mathrm{y}} \mathrm{N}$ (quantum barrier), $\mathrm{y}>\mathrm{x}$, NWs LEDs, by inserting a $20 \mathrm{~nm} \mathrm{TaN}$ interlayer in between $\mathrm{Ti}$ and silicon substrate.[5] Here we present a comparison between NWs LEDs grown on $\mathrm{Ti} / \mathrm{TaN} / \mathrm{Si}$ and $\mathrm{Ti} / \mathrm{Si}$ substrate. We achieved higher injection current and output power for the former. Our investigation opens a pathway for potential mass-production of UV LED devices utilizing existing silicon foundry.

\section{Results and discussion}
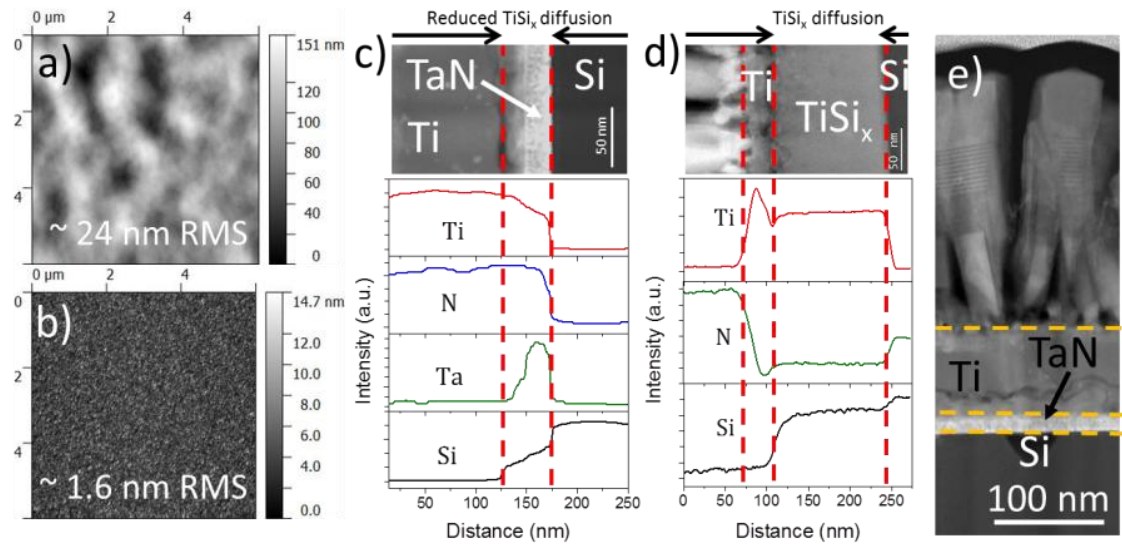

Fig. 1 (a-b) AFM map of Ti/Si and Ti/TaN/Si substrates after $600^{\circ} \mathrm{C}$ outgassing in MBE load-lock chamber prior to NWs growth. (c) STEM and EDXS line scan for the Ti/TaN/Si. (d) STEM and EELS line scan for Ti/Si sample, showing Si interdiffusion (TiSi $\mathrm{x}_{\mathrm{x}}$ ). (e) Cross-section STEM of the Ti/TaN/Si full structure.

Figure 1 (a-b) show the surface roughness comparison using atomic force microscope between the Ti/Si and $\mathrm{Ti} / \mathrm{TaN} / \mathrm{Si}$ substrates after $600{ }^{\circ} \mathrm{C}$ outgassing in the MBE load-lock chamber prior to NWs growth, noting that the as-deposited thickness are $\mathrm{Ti}=80 \mathrm{~nm}, \mathrm{Ti} / \mathrm{TaN}=80 \mathrm{~nm} / 20 \mathrm{~nm}$, respectively. An improvement in surface morphology of $16 \times$ has been achieved, reducing the RMS roughness from $24 \mathrm{~nm}$ in Ti/Si template substrate to 1.6 $\mathrm{nm}$ in Ti/TaN/Si. Figure 1 (c-d) depicts the Scanning Transmission Electron Microscopy (STEM), Electron Energy Loss Spectroscopy (EELS) and Energy Dispersive X-Ray Spectroscopy (EDXS) line-scan images of the AlGaN 
NWs LED structure on both templates. Figure 1 (c) shows significantly reduced Si interdiffusion in the Ti layer, thus allowing a flat metal starting-growth front for the NWs. On the Ti/Si platform, $80 \%$ of Ti has been converted into $\mathrm{TiSi}_{\mathrm{x}}$ in fig. 1 (d) due to silicon interdiffusion, causing NWs misorientation and potential strain-induced delamination during high injection current. Figure 1 (e) shows the STEM of a full NW structure on Ti/TaN/Si.
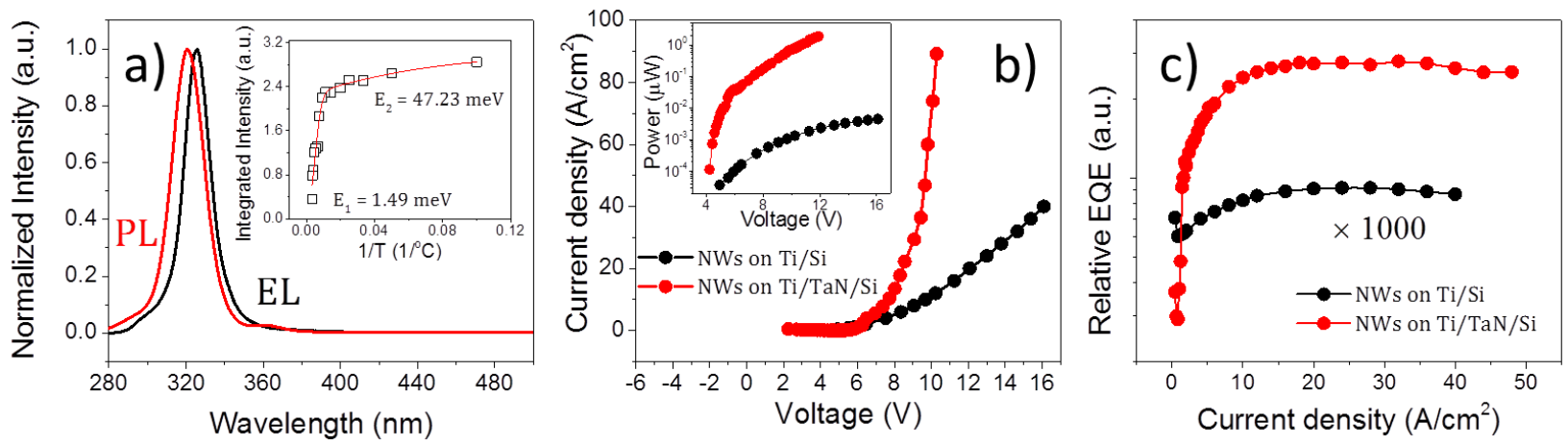

Fig. 2. (a) Photoluminescence and electroluminescence spectra (at $100 \mathrm{~mA}$ ) at $25^{\circ} \mathrm{C}$. Inset shows the Arrhenius plot and the fitted activation energies. (b) Current density vs voltage of the AlGaN NWs LEDs. Inset shows the semilog plot of optical power vs voltage. (c) Semilog plot of relative external quantum efficiencies (EQE) vs current density calculated from fig. 2 (b).

The photoluminescence (PL) and electroluminescence spectrum (EL) is depicted in fig. 2 (a) with a peak emission at 320 and $325 \mathrm{~nm}$ for the NWs LEDs grown on Ti/TaN/Si. The inset shows the Arrhenius plot and the fitted activation energies obtained from the temperature-dependent PL. The low temperature activation energy is likely due to localized states in the quantum disks, related to formation of high $\mathrm{Al}$ composition regions and to strain fluctuation. The high temperature activation energy, instead, is mainly due to thermionic emission from delocalized states (Ga-rich regions). Figure 2 (b) shows the current density vs voltage plot of the $\mathrm{Al}_{\mathrm{x}} \mathrm{Ga}_{1-\mathrm{x}} \mathrm{N} / \mathrm{Al}_{\mathrm{y}} \mathrm{Ga}_{1-\mathrm{y}} \mathrm{N} \mathrm{NWs}$ LED grown on Ti/TaN/Si and on Ti/Si. Higher current up to $90 \mathrm{~A} / \mathrm{cm}^{2}$ was achieved in this device as compared to that grown on Ti/Si substrate, despite the fact that the latter uses a more conductive $\mathrm{p}-\mathrm{GaN}$ layer while the former uses a p-AlGaN as a top non-UV-absorbing contact layer. The inset shows the output power of $1.9 \mu \mathrm{W}$ achieved at $12 \mathrm{~V}$ attributed to the use of the Ti/TaN bilayer as well as the top p-AlGaN contact layer. For completeness, figure 2 (c) shows the plot of relative external quantum efficiencies as a function of the injection current for both devices.

\section{Conclusions}

UV $\mathrm{Al}_{\mathrm{x}} \mathrm{Ga}_{1-\mathrm{x}} \mathrm{N} / \mathrm{Al}_{\mathrm{y}} \mathrm{Ga}_{1-\mathrm{y}} \mathrm{N}$ NWs LEDs were grown on Ti/TaN metal bilayer utilizing silicon substrate to eliminate the potential barrier that limits the carrier injection and heat dissipation, and to prevent device failure caused by metal delamination. We achieved higher current injection of $90 \mathrm{~A} / \mathrm{cm}^{2}$ and optical power output of $1.9 \mu \mathrm{W}$ at $12 \mathrm{~V}$. The investigation opens a pathway for reliable fabrication of UV devices on scalable substrates.

\section{References}

1. B. Janjua, H. Sun, C. Zhao, D. H. Anjum, D. Priante, A. A. Alhamoud, F. Wu, X. Li, A. M. Albadri, and A. Y. Alyamani, "Droop-free Al x Ga 1-x N/Al y Ga 1-y N quantum-disks-in-nanowires ultraviolet LED emitting at $337 \mathrm{~nm}$ on metal/silicon substrates," Optics Express 25, 1381-1390 (2017).

2. B. J. May, A. G. Sarwar, and R. C. Myers, "Nanowire LEDs grown directly on flexible metal foil," Applied Physics Letters 108, 141103 (2016).

3. H. Li, G. Zhao, L. Wang, Z. Chen, and S. Yang, "Morphology Controlled Fabrication of InN Nanowires on Brass Substrates," Nanomaterials 6, 195 (2016).

4. A. Sarwar, S. D. Carnevale, F. Yang, T. F. Kent, J. J. Jamison, D. W. McComb, and R. C. Myers, "Semiconductor Nanowire Light-Emitting Diodes Grown on Metal: A Direction Toward Large-Scale Fabrication of Nanowire Devices," Small 11, 5402-5408 (2015).

5. D. Priante, B. Janjua, A. Prabaswara, R. C. Subedi, R. T. Elafandy, S. Lopatin, D. H. Anjum, C. Zhao, T. K. Ng, and B. S. Ooi, "Highly uniform ultraviolet-A quantum-confined AlGaN nanowire LEDs on metal/silicon with a TaN interlayer," Optical Materials Express 7, 4214-4224 (2017).

\section{Acknowledgements}

We acknowledge the financial support from the King Abdulaziz City for Science and Technology (KACST), Grant No. KACST TIC R2-FP-008 and from KAUST baseline funding, BAS/1/1614-01-01. 